Rev. Mat. Iberoamericana 26 (2010), no. 2, 639-649

\title{
Lowest uniformizations of closed Riemann orbifolds
}

Rubén A. Hidalgo

\begin{abstract}
A Kleinian group containing a Schottky group as a finite index subgroup is called a Schottky extension group. If $\Omega$ is the region of discontinuity of a Schottky extension group $K$, then the quotient $\Omega / K$ is a closed Riemann orbifold; called a Schottky orbifold. Closed Riemann surfaces are examples of Schottky orbifolds as a consequence of the Retrosection Theorem. Necessary and sufficient conditions for a Riemann orbifold to be a Schottky orbifold are due to M. Reni and B. Zimmermann in terms of the signature of the orbifold. It is well known that the lowest uniformizations of a closed Riemann surface are exactly those for which the Deck group is a Schottky group. In this paper we extend such a result to the class of Schottky orbifolds, that is, we prove that the lowest uniformizations of a Schottky orbifold are exactly those for which the Deck group is a Schottky extension group.
\end{abstract}

\section{Introduction}

\subsection{Schottky extension groups}

The conformal automorphisms of the Riemann sphere $\widehat{\mathbb{C}}$ are provided by the Möbius transformations. We denote by $\mathbb{M} \cong \operatorname{PSL}(2, \mathbb{C})$ the group of Möbius transformations. The Poincaré extension theorem [10, 12] asserts that each Möbius transformation acts naturally on hyperbolic 3-space $\mathbb{H}^{3}$ as an orientation-preserving isometry. A Kleinian group is a discrete subgroup $G$ of $\mathbb{M}$. The region of discontinuity of a Kleinian group $G$ is the subset $\Omega(G)$ of $\widehat{\mathbb{C}}$ on which $G$ acts discontinuously; this is an open subset and it may be empty. The complement $\Lambda(G)=\widehat{\mathbb{C}}-\Lambda(G)$ is called the limit set of $G$. 
A function group is a pair $(F, \Delta)$, where $F$ is a finitely generated Kleinian group and $\Delta$ is an $F$-invariant connected component of $\Omega(F)$. Good sources on Kleinian groups are the books of B. Maskit [10] and of K. Matsuzaki and M. Taniguchi [12].

A Schottky group of rank $g \geq 1$ is a Kleinian group $G$ generated by loxodromic transformations $A_{1}, \ldots, A_{g}$ such that there are $2 g$ disjoint simple loops, say $C_{1}, C_{1}^{\prime}, \ldots, C_{g}, C_{g}^{\prime}$, all of them bounding a common domain $D \subset \widehat{\mathbb{C}}$, where $A_{i}\left(C_{i}\right)=C_{i}^{\prime}$, and $A_{i}(D) \cap D=\emptyset, i=1, \ldots, g$. It is well known that $\Omega(G)$ is a dense connected set, in particular, $(G, \Omega(G))$ is a function group, and that $S=\Omega(G) / G$ is a closed Riemann surface of genus $g$. The trivial group is called the Schotky group of rank $g=0$. A Schottky group of rank $g$ may also be characterized as a finitely generated and purely loxodromic Kleinian group with non-empty region of discontinuity which is isomorphic to a free group of rank $g$ [8] (another characterization of Schottky groups of rank $g$ is given by geometrically finite Kleinian groups isomorphic to free groups of rank $g$ and without parabolic transformations). As groups of hyperbolic isometries of hyperbolic space $\mathbb{H}^{3}$, Schottky groups of rank $g$ can be characterized as the universal covering groups of handlebodies of genus $g$ whose interior is endowed with a complete geometrically finite hyperbolic structure with injectivity radius bounded away from zero (we say that this is a Schottky structure on the handlebody).

A Schottky extension group is a Kleinian group containing a Schottky group as a finite index subgroup. Every finite Kleinian group is a Schottky extension group (they are exactly the ones containing a Schottky group of rank $g=0$ as finite index subgroup). Clearly, every Schottky extension group necessarily contains a Schottky group as a finite index normal subgroup. Let $K$ be a Schottky extension group and let $G$ be a Schottky group, say of rank $g$, which is a finite index normal subgroup of $K$. Let $M=\mathbb{H}^{3} / G$ be the handlebody with the Schottky structure provided by the Schottky group $G$. As $G$ is a normal subgroup of finite index in $K$, it follows that $K$ induces a finite group, isomorphic to $K / G$, of orientation-preserving isometries of the interior of $M$. Conversely, the lifting to the universal cover $\mathbb{H}^{3}$ of a finite group of orientation-preserving isometries of the interior of $M$, with a Schottky structure produced by a Schottky group $G$, produces a Kleinian group $K$ containing $G$ as a normal subgroup of finite index, that is, a Schottky extension group.

\subsection{Closed Riemann orbifolds}

A closed Riemann orbifold is a pair $\mathcal{O}=(S, \mathcal{P})$, where $S$ is a closed Riemann surface, called the underlying Riemann surface structure of $\mathcal{O}$, and $\mathcal{P}$ is a finite collection (maybe empty) of pairs $\left(p_{j}, n_{j}\right)$, where $p_{j} \in S$ are pairwise 
different, called the conical points, and $n_{j} \geq 2$ is an integer, called the order of $p_{j}$. The signature of $\mathcal{O}$ is the tuple $\left(g ; n_{1}, \ldots, n_{r}\right)$, where $g$ is the genus of $S$ and $r$ is the cardinality of $\mathcal{P}$. We say that $\mathcal{O}$ is of hyperbolic type if $2 g-2+\sum_{j=1}^{r}\left(1-1 / n_{j}\right)>0$. The orbifold fundamental group of $\mathcal{O}$ is

$\pi_{1}^{\mathrm{orb}}(\mathcal{O})=\left\langle a_{1}, \ldots, a_{g}, b_{1}, \ldots, b_{g}, c_{1}, \ldots, c_{r} ; c_{1}^{n_{1}}=\cdots=c_{r}^{n_{r}}=1=\prod_{j=1}^{g}\left[a_{j}, b_{j}\right] \prod_{i=1}^{r} c_{i}\right\rangle$,

where $[a, b]=a b a^{-1} b^{-1}$. Note that if $\mathcal{P}=\emptyset$, then we identify the Riemann orbifold $\mathcal{O}$ with the Riemann surface $S$. Two closed Riemann orbifolds, say $\mathcal{O}_{1}=\left(S_{1}, \mathcal{P}_{1}\right)$ and $\mathcal{O}_{2}=\left(S_{2}, \mathcal{P}_{2}\right)$, are said to be conformally equivalent if there is a conformal homeomorphism $h: S_{1} \rightarrow S_{2}$ inducing an ordering preserving bijection of conical points. A good source about orbifolds is, for instance, the notes of W.P. Thurston [16].

\subsection{Riemann orbifolds from function groups}

Let $(F, \Delta)$ be a function group so that $\Delta / F$ is closed. It follows from Ahlfors finiteness theorem [1] that $F$ is finitely generated.

The quotient $\Delta / F$ defines naturally a closed Riemann orbifold. The conical points are the projections of those points $z \in \Delta$ with non-trivial stabilizer $F_{z}<F$ (a cyclic subgroup) and the corresponding orders are the orders of $F_{z}$. The complement $S_{0}$ in $\Delta / F$ of the above conical points has a natural structure of a Riemann surface, this being the complement of a finite number points of a closed Riemann surface, say $S$. By the results in [11], the Riemann surface structure of $S$ is unique up to conformal equivalence. The underlying Riemann surface structure of the Riemann orbifold is $S$.

By the Selberg lemma [3, 15], the group $F$ has a finite index normal torsion free subgroup $N$. In this way, $R=\Delta / N$ is a closed Riemann surface and $H=F / N$ is a finite group of conformal automorphisms of $R$ so that $\Delta / F=R / H$.

\subsection{Good Riemann orbifolds}

A closed Riemann orbifold is called a good orbifold if it is obtained as the quotient of a closed Riemann surface by a finite group of conformal automorphisms; otherwise, it called a bad orbifold. The only signatures corresponding to bad orbifolds are: $(i)(0 ; n), n \geq 2$, (ii) $(0 ; n, m), n \neq m$. As already noted above, the Riemann orbifold produced by a function group is a good orbifold. From now on in this paper, an orbifold will mean a good orbifold. 


\subsection{Uniformizations}

An uniformization of a closed Riemann orbifold $\mathcal{O}=(S, \mathcal{P})$ is a triple $(\Delta, F, Q: \Delta \rightarrow S)$ so that:

(i) $(F, \Delta)$ is a function group,

(ii) $Q: \Delta \rightarrow S$ is a holomorphic (branched) covering map with $\operatorname{Deck}(Q)=F$,

(iii) the conical points of $\mathcal{O}$ are exactly the branched values of $Q$, and

$(i v)$ the orders of conical points are equal to the orders of the branch values of $Q$.

Note that if $\mathcal{P}=\emptyset$, the above definition corresponds to the usual notion of uniformization of closed Riemann surfaces.

If $G$ is a Schottky group and $S=\Omega(G) / G$, then $(\Omega(G), G, Q: \Omega(G) \rightarrow S)$ is called a Schottky uniformization of $S$. As a consequence of the retrosection theorem (e.g. $[4,6]$ ), every closed Riemann surface has a Schottky uniformization.

Let $\mathcal{O}=(S, \mathcal{P})$ be a closed Riemann orbifold of a hyperbolic type. It follows from the Klein-Poincaré uniformization theorem [13] the existence of a co-compact Fuchsian group $\Gamma \cong \pi_{1}^{\text {orb }}(\mathcal{O})$, acting on the hyperbolic plane $\mathbb{H}^{2}$, so that the closed Riemann orbifold induced by $\mathbb{H}^{2} / \Gamma$ is conformally equivalent to $\mathcal{O}$. The uniformization $\left(\mathbb{H}^{2}, \Gamma, P: \mathbb{H}^{2} \rightarrow S\right)$ is called a Fuchsian uniformization.

The classification of uniformizations of closed Riemann surfaces is provided by the following result due to B. Maskit [9].

Theorem 1 (Classification of Uniformizations [9]). Let $S$ be a closed Riemann surface.

(1) If $(\Delta, G, Q: \Delta \rightarrow S)$ is an uniformization of $S$, then there exists a collection $\left\{\alpha_{m}\right\}$ of (homotopically independent) pairwise disjoint simple loops on $S$ and a collection of positive integers $\left\{k_{m}\right\}$ so that $Q: \Delta \rightarrow S$ is a regular covering for which

(1.1) each of the loops $w_{m}=\alpha_{m}^{k_{m}}$ lifts to a loop, and

(1.2) every loop in $\Delta$ is freely homotopic to a product of the liftings of these loops.

(2) Given any collection $\left\{\alpha_{m}\right\}$ of (homotopically independent) pairwise disjoint simple loops on $S$ and a collection of positive integers $\left\{k_{m}\right\}$, there is an uniformization $(\Delta, G, Q: \Delta \rightarrow S)$ of $S$ so that $Q: \Delta \rightarrow S$ satisfies $(1.1)$ and $(1,2)$ above.

We say that the set of loops, $\left\{w_{m}=\alpha_{m}^{k_{m}}\right\}$, determines the covering $Q: \Delta \rightarrow S$. 
In Theorem 1, the Schottky uniformizations are exactly the ones for which $k_{m}=1$, for every $m$, and the collection of simple loops $\left\{\alpha_{m}\right\}$ cutoff $S$ into planar surfaces.

\subsection{Schottky extension uniformizations}

Let $K$ be a Schottky extension group with $\Omega=\Omega(K)$. Let $\mathcal{O}=(S, \mathcal{P})$ be the natural closed Riemann orbifold defined by $\Omega / K$ and let $Q: \Omega \rightarrow S$ be the natural factor map. Then the uniformization $(\Omega, K, Q: \Omega \rightarrow S)$ is called a Schottky extension uniformization of $\mathcal{O}$.

A closed Riemann orbifold admitting a Schottky extension uniformization will be called a Schottky orbifold. In [14] M. Reni and B. Zimmermann have provided necessary and sufficient conditions for a closed Riemann orbifold to be a Schottky one in terms of the signature of the orbifold.

Proposition 2 ([14]). Let $\mathcal{O}$ be a closed Riemann orbifold of a hyperbolic type with the signature $\left(g ; n_{1}, \ldots, n_{r}\right)$. Then $\mathcal{O}$ is a Schottky orbifold if and only if one of the following conditions holds.

(i) $g>0$.

(ii) The (unordered) numbers $n_{1}, \ldots, n_{r}$ occur in pairs.

(iii) Among the numbers $n_{1}, \ldots, n_{r}$ there are at least two disjoint occurrences out of the following four situations: $(2),(3,3),(3,4)$, or $(3,5)$ (possible two times the same).

Another necessary and sufficient condition for a closed Riemann orbifold (not necessarily of a hyperbolic type) is provided by the following.

Proposition 3. The Schottky orbifolds $\mathcal{O}$ are exactly those orbifolds obtained as quotients $R / H$, where $R$ is a closed Riemann surface, $H$ is a finite group of conformal automorphisms of $R$ and there is a Schottky uniformization $(\Omega, G, P: \Omega \rightarrow R)$ of $R$ so that $H$ lifts, that is, for each $h \in H$ there is a conformal automorphism $\widehat{h}$ of $\Omega$ so that $h \circ P=P \circ \widehat{h}$.

Proof. Let $\mathcal{O}=(S, \mathcal{P})$ be a closed Riemann orbifold. Let $R$ be a closed Riemann surface and $H$ be a finite group of conformal automorphisms of $R$ so that $\mathcal{O}$ is defined by $R / H$. Assume there is a Schottky uniformization $(\Omega(G), G, P: \Omega(G) \rightarrow R)$ of $R$ so that $H$ lifts. By lifting $H$, with respect to this Schottky uniformization, we obtain a group $K$ of conformal automorphisms of $\Omega(G)$ containing $G$ as a finite index normal subgroup. It is known that $\Omega(G)$ is of class $O_{A D}$; that is, it admits no holomorphic function with finite Dirichlet norm (see [2, pg. 241]). It follows from this (see $[2$, pg. 200]) that every conformal map from $\Omega(G)$ into the Riemann 
sphere is a Möbius transformation. In this way, $K$ is a Kleinian group and, in particular, a Schottky extension group. By the construction, the orbifold $\mathcal{O}$ is uniformized by $K$, so $\mathcal{O}$ is a Schottky orbifold. Conversely, let us assume that $\mathcal{O}$ is a Schottky orbifold and consider a Schottky extension uniformization $(\Omega, K, P: \Omega \rightarrow S)$. As already noted before, $K$ contains, as a finite index normal subgroup, a Schottky group $G$. As a finite index subgroup of $K$ has the same region of discontinuity $\Omega$, it is enough to take $R=\Omega / G$ and $H=K / G$.

As a consequence of Proposition 2 (also by Proposition 3 and the results in [5]), the orbifolds with signature of the form $(0 ; a, b, c)$ are not Schottky orbifolds.

We will need the following lifting criteria of conformal automorphisms of closed Riemann surfaces to their uniformizations. This criteria concerns the existence of an invariant (with respect to the group of automorphisms) collection of pairwise disjoint simple loops defining the uniformization.

Theorem $4([5])$. Let $(\Delta, F, P: \Delta \rightarrow S)$ be an uniformization of a closed Riemann surface $S$, of genus $g \geq 2$, and let $F$ be torsion free. Let $H$ be a group of (conformal/anticonformal) automorphism of $S$. Then the group $H$ lifts, with respect to $(\Delta, F, P: \Delta \rightarrow S)$, if and only if there is a collection $\mathcal{F}$ of pairwise disjoint simple loops on $S$ defining the covering $P: \Delta \rightarrow S$ which is invariant under the action of $H$.

\subsection{Lowest uniformizations}

The collection of uniformizations of an orbifold $\mathcal{O}=(S, \mathcal{P})$ is partially ordered. We say that an uniformization $\left(\Delta_{1}, G_{1}, P_{1}: \Delta_{1} \rightarrow S\right)$ of $\mathcal{O}$ is higher than an uniformization $\left(\Delta_{2}, G_{2}, P_{2}: \Delta_{2} \rightarrow S\right)$ of $\mathcal{O}$ if there is a (necessarily holomorphic) covering map $T: \Delta_{1} \rightarrow \Delta_{2}$ so that $P_{1}=P_{2} \circ T$. Equivalently, there is a normal subgroup $K$ of $G_{1}$ and an uniformization $\left(\Delta_{1}, K, T: \Delta_{1} \rightarrow \Delta_{2}\right)$ of $\Delta_{2}$ so that $P_{1}=P_{2} \circ T$.

The highest uniformizations of $\mathcal{O}=(S, P)$ are provided by universal coverings, that is, by the uniformizations $(\Delta, F, P: \Delta \rightarrow S)$ of $\mathcal{O}$ for which $\Delta$ is simply-connected. If $\mathcal{O}$ is of a hyperbolic type, then highest uniformizations are (equivalent to) Fuchsian ones.

It follows from the defined order that an uniformization $(\Delta, G, P: \Delta \rightarrow$ $S$ ) of the orbifold $\mathcal{O}$ will be a lowest one if there is no non-trivial normal subgroup $N$ of $G$ acting freely on $\Delta$ and with $\Delta / N$ a planar surface.

It is a well known fact [9] that the lowest uniformizations of closed Riemann surfaces are given by the Schottky uniformizations. The main result of this paper is the following description of the lowest uniformizations of the Schottky orbifolds. 
Theorem 5. The lowest uniformizations of Schottky orbifolds are given by Schottky extensions uniformizations.

\section{Proof of Theorem 5}

\subsection{First part}

First, we prove that Schottky extension uniformizations are lowest ones.

Let us consider a Schottky extension uniformization of a Schottky orbifold $\mathcal{O}=(S, \mathcal{P})$, say $(\Omega, K, P: \Omega \rightarrow S)$; in which case $\Omega=\Omega(K)$. Let $G$ be a Schottky group, say of rank $g$, which is a finite index normal subgroup of $K$; so the region of discontinuity of $G$ is again $\Omega$. Let us consider the Schottky uniformization $(\Omega, G, L: \Omega \rightarrow R$ ), where $R=\Omega / G$.

Let us assume our Schottky extension uniformization is not a lowest one. It follows that there exists a normal subgroup $N$ of $K, N \neq K$, so that $\Delta=\Omega / N$ is planar and has no conical points (this means that $N$ acts on $\Omega$ without fixed points).

If $g=0$, then $G$ is the trivial group and $K$ is necessarily a finite group and $\Omega=\widehat{\mathbb{C}}$. As any finite group of Möbius transformations, different from the trivial group, acts with fixed points on $\widehat{\mathbb{C}}$, it follows that $N$ acts with fixed points on $\Omega$, a contradiction.

Let us now assume $g \geq 1$. We may assume, by the Planarity Theorem [9], that $\Delta$ is a domain in the Riemann sphere and that $\Gamma=K / N$ acts as a Kleinian group. In this way, there is an uniformization $(\Delta, \Gamma, Q: \Delta \rightarrow S)$ of the orbifold $\mathcal{O}$ and there is an uniformization $(\Omega, N, T: \Omega \rightarrow \Delta)$ of the planar surface $\Delta$ so that $P=Q \circ T$.

Clearly, $N \cap G$ is a normal subgroup of $K$. As $\Omega / N$ is a planar Riemann surface, the quotient $\Sigma=\Omega /(N \cap G)$ must also be a planar Riemann surface. Again, using the Planarity Theorem [9], we may assume that $\Sigma$ is a domain in the Riemann sphere and that $H=G /(N \cap G)$ acts as a Kleinian group. There is a uniformization $(\Sigma, H, V: \Sigma \rightarrow R)$ and there is a uniformization $(\Omega, G \cap N, U: \Omega \rightarrow \Sigma)$ so that $L=V \circ U$. As the Schottky uniformization $(\Omega, G, L: \Omega \rightarrow R)$ is a lowest one, we must have $N \cap G=G$, in which case $\Sigma$ is a non-planar surface, a contradiction.

\subsection{Second part}

Secondly, we proceed to prove that every lowest uniformization of a Schottky orbifold is a Schottky extension one.

Let us consider a lowest uniformization $(\Delta, K, P: \Delta \rightarrow S)$ of the orbifold $\mathcal{O}=(S, \mathcal{P})$. By the Selberg lemma [15], there is a torsion free finite index 


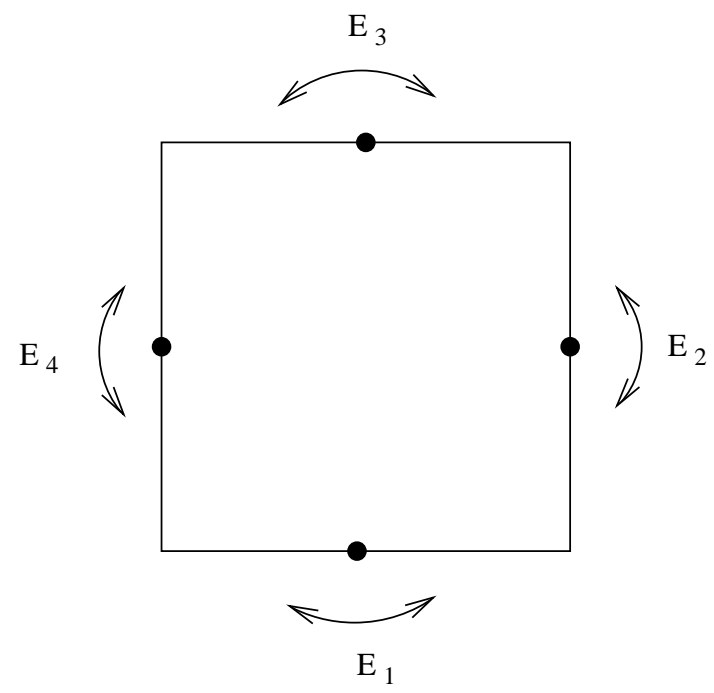

Figure 1: Fundamental domain for $K$

normal subgroup $G$ of $K$. Set $R=\Delta / G$, which is a closed Riemann surface of genus $g$.

If $g=0$, then $G$ is the trivial group and $K$ is a finite group of Möbius transformations; so a Schottky extension group.

If $g=1$, then $G$ is either $(i)$ a Schottky group of rank 1, or (ii) an Abelian group generated by two parabolic transformations with a common fixed point. In case $(i)$ we have that $K$ is a Schottky extension group. In case $(i i)$ we are in presence of a universal covering of $R$ and $\Delta=\mathbb{C}$. In this last case, the only possibility for $\Delta / K$ to be a Schottky orbifold is to be of signature $(0 ; 2,2,2,2)$ (by Theorem 2 and checking the possible orbifold quotients regularly covered by tori). In this case, as can be seen in the book [10], $K$ is generated by 4 elliptic elements of order 2 , say $E_{1}, \ldots, E_{4}$, so that $E_{4} E_{3} E_{2} E_{1}=1$ (see Figure 1 ). The cyclic subgroup $J$, generated by the parabolic transformation $E_{3} E_{1}$, is a normal subgroup of $K$ so that $K / J$ is isomorphic to the free product $\mathbb{Z}_{2} \star \mathbb{Z}_{2}$ whose topological action of $K / J$ is equivalent to the one given in Figure 2, we get a contradiction to the fact that $K$ produces a lowest uniformization.

Assume now on that $g \geq 2$. By construction, there is an uniformization of $R$, say $(\Delta, G, Q: \Delta \rightarrow R)$, and a (branched) regular covering map $T$ : $R \rightarrow S$ with $\operatorname{Deck}(T)=K / G$, a finite group of conformal automorphisms of $R$, so that $T \circ Q=P$. Set $H=K / G$. As the group $H$ lifts, with respect to the uniformization $(\Delta, G, Q: \Delta \rightarrow R)$, Theorem 4 asserts that there is a collection $\mathcal{F}$ of pairwise disjoint simple loops on $R$ which is invariant under the action of $H$ and defines the regular planar covering $Q: \Delta \rightarrow R$. 
Lemma 6 below asserts that $(\Delta, G, Q: \Delta \rightarrow R)$ is a lowest uniformization of the closed Riemann surface $R$, so it is a Schottky uniformization. It follows that $G$ is a Schottky group and that $K$ is an extended Schottky group as desired.

Lemma 6. $(\Delta, G, Q: \Delta \rightarrow R)$ is a lowest uniformization.

Proof. In fact, if $(\Delta, G, Q: \Delta \rightarrow R)$ is not a lowest uniformization, then we may add extra simple loops to $\mathcal{F}$ in order to obtain a maximal collection $\mathcal{N}$ of pairwise disjoint simple loops which is invariant under $H$. In this case, the new collection $\mathcal{N}$ produces a Schottky uniformization of $R$ for which $H$ lifts. Let this Schottky uniformization be $(\Omega, F, L: \Omega \rightarrow R)$. There is also an uniformization $(\Delta, N, V: \Delta \rightarrow \Omega)$ so that $Q=L \circ V$. At this point, we have that $N$ is a normal subgroup of $G$. Now, by the lifting property of $H$ with respect to this Schottky uniformization and the $O_{A D}$ property of $\Omega[2]$, there is a Kleinian group $\widehat{H}$ containing $F$ as a finite index normal subgroup and so that $H=\widehat{H} / F$. It follows that $N$ is also a normal subgroup of $K$ so that $K / N=\widehat{H}$. This asserts that $(\Delta, K, P: \Delta \rightarrow S)$ is not a lowest uniformization of $\mathcal{O}$, a contradiction.

\section{Final remarks}

\subsection{More general Riemann orbifolds}

Theorem 5 describes the lowest uniformizations of the Schottky orbifolds. One may ask for a description of the lowest uniformizations of the rest of closed Riemann orbifolds. We already noted that an orbifold $\mathcal{O}=(S, \mathcal{P})$ of signature of the form $(0 ; a, b, c)$ is not of Schottky type. In this case, an uniformization $(\Delta, K, P: \Delta \rightarrow S)$ corresponds to a function group $(\Delta, K)$ so that $\Delta / K$ describes $\mathcal{O}$. As consequence of results in [7], it happens that such a function group is in fact a Fuchsian group; that is, the only uniformizations of such an orbifold is given by the Fuchsian ones. By Theorem 2, the only closed Riemann orbifolds we are left to study are the hyperbolic ones of genus zero which are not of Schottky type.

\subsection{Topological types}

Schottky groups providing uniformizations of closed Riemann surfaces of the same genus are topologically equivalent, but there are examples of topologically different Schottky extension groups providing uniformizations of topologically equivalent Schottky orbifolds. For instance, let $K_{1}$ be the free 

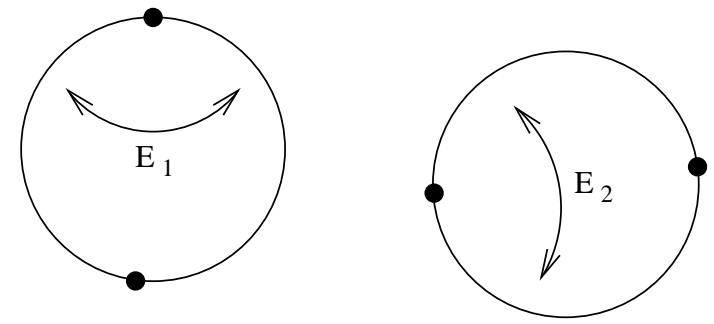

Figure 2: Fundamental domain for $K_{1}$

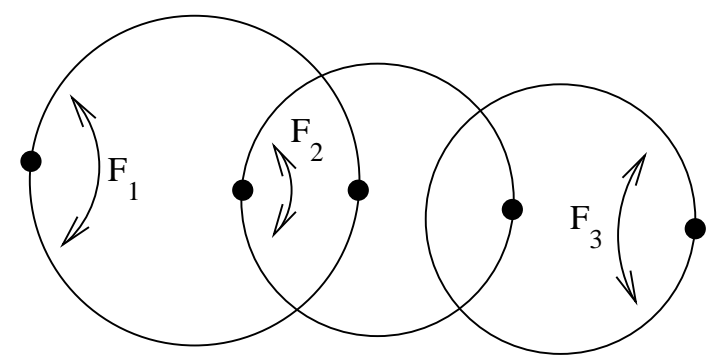

Figure 3: Fundamental domain for $K_{2}$. The circles intersect orthogonally.

product of two elliptic transformations of order 2, say $E_{1}$ and $E_{2}$ (see Figure 2), and let $K_{2}$ be generated by three elliptic transformations of order 2, say $F_{1}, F_{2}$ and $F_{3}$ so that $\left(F_{2} F_{1}\right)^{2}=\left(F_{3} F_{2}\right)^{2}=1$ (see Figure 3 ). Both Kleinian groups are Schottky extension groups, in fact, $K_{1}$ contains as an index two subgroup the Schottky group $G_{1}=\left\langle A_{1}=E_{2} E_{1}\right\rangle$ and $K_{2}$ contains as an index two subgroup the Schottky group $G_{2}=\left\langle A_{2}=F_{3} F_{1}\right\rangle$. Also, both Kleinian groups $K_{1}$ and $K_{2}$ provide uniformizations of orbifolds with the signature $(0 ; 2,2,2,2)$ and they are clearly non-isomorphic as abstract groups; in particular, they cannot be topologically conjugated.

Acknowledgments. The author wishes to express his special thanks to the Referee for his/her careful reading and the many suggestions and corrections. Also, the author thanks Ara Basmajian for the useful comments and proofreading done to the previous version of this paper.

\section{References}

[1] Ahlfors, L.: Finitely generated Kleinian groups. Amer. J. Math. 86 (1964), 413-429. Correction ibid 87 (1965), 759.

[2] Ahlfors, L. and Sario, L.: Riemann Surfaces. Princeton Mathematical Series 26. Princeton University Press, Princeton, NJ, 1960.

[3] Alperin, R. C.: An elementary account of Selberg's lemma. Enseign. Math. (2) 33 (1987), no. 3-4, 269-273. 
[4] Bers, L.: Automorphic forms for Schottky groups. Advances in Math. 16 (1975), 332-361.

[5] Hidalgo, R. A.: Automorphisms groups of Schottky type. Ann. Acad. Sci. Fenn. Math. 30 (2005), no. 1, 183-204.

[6] Koebe, P.: Über die Uniformisierung der algebraischen Kurven II. Math. Ann. 69 (1910), no. 1, 1-81.

[7] KrA, I.: Deformations of Fuchsian groups, II. Duke Math. J. 38 (1971), 499-508.

[8] Maskit, B.: A characterization of Schottky groups. J. Analyse Math. 19 (1967), 227-230.

[9] Maskit, B.: A theorem on planar covering surfaces with applications to 3-manifolds. Ann. of Math. (2) 81 (1965), 341-355.

[10] Maskit, B.: Kleinian groups. Grundlehren der Mathematischen Wissenschaften 287. Springer-Verlag, Berlin, 1988.

[11] Maskit, B.: Canonical domains on Riemann surfaces. Proc. Amer. Math. Soc. 106 (1989), no. 3, 713-721.

[12] Matsuzaki, K. And Taniguchi, M.: Hyperbolic manifolds and Kleinian groups. Oxford Mathematical Monographs. Oxford Science Publications. The Clarendon Press, Oxford University Press, New York, 1998.

[13] Poincaré, H.: Sur l'uniformisation des fonctions analytiques. Acta Math. 31 (1908), no. 1, 1-64.

[14] Reni, M. and Zimmermann, B.: Handlebody orbifolds and Schottky uniformizations of hyperbolic 2-orbifolds. Proc. Amer. Math. Soc. 123 (1995), no. $12,3907-3914$.

[15] SelBerG, A.: On discontinuous groups in higher-dimensional symmetric spaces. In Contributions to function theory, 147-164. Tata Institute of Fundamental Research, Bombay, 1960.

[16] Thurston, W.P.: The geometry and topology of 3-manifolds. Mimeographed notes. Princeton, 1979.

Recibido: 26 de septiembre de 2008

Revisado: 23 de febrero de 2009

Rubén A. Hidalgo

Departamento de Matemática

Universidad Técnica Federico Santa María

Casilla 110-V Valparaiso, Chile

ruben.hidalgo@usm.cl

The author is partially supported by projects Fondecyt 1070271 and UTFSM 120801. 